\title{
Recent extensions to GALPROP
}

\section{A. W. Strong*}

Max-Planck-Institut für extraterrestrische Physik

E-mail: aws @mpe.mpg.de

Some recent extensions to the GALPROP cosmic-ray propagation package are described. The enhancements include: an accurate solution option, improved convection formulation, alternative spatial boundary conditions, polarized synchrotron emission, new magnetic field models, updated gamma-ray production cross-sections, free-free radio emission and absorption, primary positrons, additional injection spectral breaks, deuterium production by pp fusion, hadronic energy losses, improved HEALPix skymap format, compatibility with latest HEALPix release, and various bug fixes. The Explanatory Supplement has been extensively updated, including details of these enhancements. A compatible plot package GALPLOT for GALPROP output is also provided, as well as other related software.

The 34th International Cosmic Ray Conference,

30 July- 6 August, 2015

The Hague, The Netherlands

\footnotetext{
* Speaker.
} 


\section{Introduction}

GALPROP is a software package for numerical Galactic cosmic-ray propagation and related emission processes. Its origins go back more than 20 years $(1 ; 2 ; 3)$. The first mature version of GALPROP was presented in $(4 ; 5)$. and it has been developed further in the following years. It is described in a wider context in (6). A recent related review is (7).

The extensions described here were made based on the C++ version (v54) publicly released four years ago in $2011^{1}$. These developments were prompted by new requirements and features which appeared desirable, including suggestions by users. The extended version is available ${ }^{2}$; it has been downloaded over 400 times since its initial release in 2013, showing the community interest. It is an ongoing development, with regular updates. GALPROP is maintained under the configuration control system subversion ( $s v n$ ). I briefly describe each of the new features, grouped according to topic, with desiderata for future developments. Details can be found in the Explanatory Supplement document included in the package.

\section{Physical processes}

\subsection{Hadronic production cross-sections}

There has been progress in hadronic $\gamma$-ray production physics $(8 ; 9 ; 10)$ and this is reflected in new options including combinations appropriate to low and high energy protons ${ }^{3}$. A correction for nuclei heavier than Helium in both CR and interstellar gas is provided. Corresponding options for secondary positrons and electrons are foreseen in future.

\subsection{Hadronic energy losses}

Energy loss of nuclei by hadronic processes (pion production) was not included previously, only ionization and Coulomb losses were present, so that energy conservation was not respected since there is hadronic production of $\gamma$-rays and leptons. Hadronic losses are comparable to ionization and Coulomb losses around $1 \mathrm{GeV}$. This is a potentially important process especially in regions of high gas density. It has been included in a simple treatment based on $(11 ; 12)$ for protons and Helium. This is a complex problem however, and further developments are desirable. In particular how to treat losses in the context of nuclear fragmentation, beyond the approximation of conserved energy per nucleon, is difficult.

\subsection{Deuterium production by pp fusion}

At energies up to $1 \mathrm{GeV}$, deuterium production by pp fusion is significant and has been included $^{4}$ using the formulation in (13). See also Picot-Clemente et al. this conference, paper PoS(ICRC2015)555.

\footnotetext{
${ }^{1}$ http://galprop.stanford.edu. Last update 2011.

${ }^{2}$ https://sourceforge.net/projects/galprop

${ }^{3}$ Thanks to Chuck Dermer, Michael Kachelriess and Sergey Ostapchenko for providing the relevant code, and much help on this topic.

${ }^{4}$ Thanks to Nicolas Picot-Clemente for suggesting this topic, and help in its implementation.
} 


\subsection{Synchrotron radiation}

The treatment of synchrotron radiation from electrons and positrons has been updated to include polarization using the regular component of the magnetic field. For a detailed description and results see $(14 ; 15)$. GALPROP models of the synchrotron spectral index have been used in the analysis of Galactic microwave emission observed by Planck (16).

\subsection{Free-free emission and absorption}

Although not a CR-related process, radio surveys include free-free emission from thermal electrons, so skymaps are now produced. These are relevant at frequencies above $1 \mathrm{GHz}$, and dominated for WMAP and Planck. The model is based on the NE2001 thermal electron model. Free-free absorption is important at radio frequencies below $100 \mathrm{MHz}$, and this can be applied to both synchrotron and free-free emission. Again NE2001 is used. See (15) for details and results. Developments beyond NE2001 would be desirable in future since this has known problems.

\section{Cosmic-ray propagation}

\subsection{Accurate solution options}

The original numerical scheme for solving the CR propagation equations uses a Crank-Nicolson scheme with operator splitting, and a procedure to accelerate the solution by varying the time step from large to small values. Both the operator splitting and time-reduction schemes have the effect that the true solution to the steady-state equation is not obtained (see Explanatory Supplement and also (17)). This can be checked by detailed diagnostics of the solution, provided as a GALPROP option. In order to obtain accurate solutions, an explicit scheme is now available, which uses small enough time-steps that none of the mentioned approximations are required, and demonstrably converges to the steady-state solution. Detailed analysis is in the Explanatory Supplement. The disadvantage of this method is the long computational time on account of the small time steps. This is clearly a brute-force approach, and the application of modern numerical methods, such as in PICARD (18; 17), which can solve the equations fast and accurately, would be preferable in future.

\subsection{Anisotropic diffusion}

Spatial diffusion was assumed to be isotropic, but theory predicts that it is anisotropic, being slower perpendicular to the regular field, hence slower in the $z$-direction. An option for separate diffusion coefficients in the Galactic plane and perpendicular to it has therefore been implemented.

In future, a more specific relation to the regular B-field would be desirable.

\subsection{Convective transport}

Convection (Galactic wind) was originally represented as a velocity increasing linearly from the Galactic plane, which is rather unphysical. A more plausible form has been introduced, which still respects the condition of zero velocity at $z=0$ (since the sign must reverse there) and which smoothly increases to a constant value beyond some $z$. The form (using a tanh function) is controlled by two parameters for the strength and $z$-dependence of the wind. In future a physical description of the wind would be desirable. 


\subsection{Boundary conditions}

The spatial boundary condition imposed has been zero CR density at all boundaries in $2 \mathrm{D}$ $(R, z)$ or 3D $(x, y, z)$. This is clearly not physical since even with free-escape, the density is non-zero there. The condition was imposed by simply forcing a zero value after each time-step, rather than acually solving with this condition. As an alternative, the boundary condition is not imposed, so particles flow out but not back in at the boundary. The density at the boundary is non-zero. A more physical approach actually handling the physics of the boundary, would be desirable in future.

\subsection{Primary positrons}

There is good experimental evidence for a primary component of positrons (PAMELA, AMS02), in addition to secondaries from hadronic interactions. Previously only secondary positrons were included. Primary positrons can now be included, with an injection spectrum and spatial source distribution independent of primary electrons. Note that primary positrons also contribute to $\gamma$-rays via inverse-Compton and bremsstrahlung, and to synchrotron emission, so are important to take into account.

\subsection{Injection spectrum}

Additional spectral breaks in the injection spectra of nuclei, electrons and positrons are now available, for more flexibility in fitting observations.

\section{Galactic structure}

\subsection{Magnetic fields}

Additional models of the Galactic magnetic field, including the regular field described by (19; 20), are now included.

\section{Format and examples}

\subsection{HEALPix}

Skymap output ( $\gamma$-rays , radio) is provided in HEALPix (21) as the format of choice for fullsky coverage with uniform pixel size. New versions of HEALPix have become available ${ }^{5}$, and the GALPROP code has been adapted to enable their use. The format has been adjusted so that the energy (or frequency for radio) is stored as FITS columns rather than the original format of vectors within pixels. This is compatible with the Aladin visualization package ${ }^{6}$, and also more convenient for reading by user software.

\subsection{Examples and reference output}

A variety of example parameter files (galdef files) are provided to illustrate the many features including the new ones. As an installation check, a full set of output files is also provided for one sample case.

\footnotetext{
${ }^{5}$ http://healpix.jpl.nasa.gov

${ }^{6}$ http://aladin.u-strasbg.fr
} 


\subsection{Bug fixes}

Various (minor) bug fixes to the original version were made during the development.

\section{Related software}

A plotting package GALPLOT, for cosmic rays, $\gamma$-rays and radio, compatible with GALPROP output, is now available ${ }^{7}$. It is compatible with the cosmic-ray database CRDB (22), and can handle Fermi-LAT and other $\gamma$-ray data and various synchrotron surveys. GALPLOT can also be used for Galactic source population synthesis, as described in section 6 of (23).

Separate routines for computing synchrotron ${ }^{8}$, anisotropic inverse Compton scattering, and models for inverse-Compton emission from solar and stellar heliospheres are also available ${ }^{9}$

\section{Outlook}

Other projects complementary to GALPROP are in progress. DRAGON (24) includes various new features. PICARD $(18 ; 17)$ allows full 3D models with high resolution using advanced numerical methods. The semi-analytical USINE (25) package is becoming public (see Maurin, this conference, paper ID 296). These activities will stimulate further GALPROP developments.

\section{References}

[1] A. W. Strong and G. Youssefi, Spectrum of electrons with inhomogeneous energy losses, Proc. 23rd ICRC 2 (1993) 124-127,

[http://www.mpe.mpg.de/ aws/publications/calgaryprop.ps].

[2] A. W. Strong and G. Youssefi, Propagation models for cosmic-ray nucleons and electrons and predictions of the Galactic gamma-ray spectrum, Proc. 24th ICRC 3 (1995) 48-51, [http://www.mpe.mpg.de/ aws/publications/strong_youssefi_icrc_24.ps].

[3] A. W. Strong and G. Youssefi, Cosmic-ray propagation: self-consistent models for nuclei, electrons and gamma rays, http://www.mpe.mpg.de/ aws/publications/propagate_paper1.ps.

[4] I. V. Moskalenko and A. W. Strong, Production and Propagation of Cosmic-Ray Positrons and Electrons, ApJ 493 (Jan., 1998) 694-+.

[5] A. W. Strong and I. V. Moskalenko, Propagation of Cosmic-Ray Nucleons in the Galaxy, ApJ 509 (Dec., 1998) 212-228.

[6] A. W. Strong, I. V. Moskalenko, and V. S. Ptuskin, Cosmic-Ray Propagation and Interactions in the Galaxy, Annual Review of Nuclear and Particle Science 57 (2007) 285-327, [astro-ph/].

\footnotetext{
${ }^{7}$ https://sourceforge.net/projects/galplot

${ }^{8} \mathrm{https} / / /$ sourceforge.net/projects/galpropsynchrotron

${ }^{9} \mathrm{https} / / /$ sourceforge.net/projects/stellarics
} 
[7] I. A. Grenier, J. H. Black, and A. W. Strong, The Nine Lives of Cosmic Rays, Ann. Rev. Astron. Astrophys. 53 (2015) 199-246, [doi:10.1146/annurev-astro-082214-122457].

[8] C. D. Dermer, A. W. Strong, E. Orlando, L. Tibaldo, and for the Fermi Collaboration, Determining the Spectrum of Cosmic Rays in Interstellar Space from the Diffuse Galactic Gamma-Ray Emissivity, ArXiv e-prints (July, 2013) [arXiv: 1307.0497].

[9] C. D. Dermer, J. D. Finke, R. J. Murphy, A. W. Strong, F. Loparco, M. N. Mazziotta, E. Orlando, T. Kamae, L. Tibaldo, J. Cohen-Tanugi, M. Ackermann, T. Mizuno, and F. W. Stecker, On the Physics Connecting Cosmic Rays and Gamma Rays: Towards Determining the Interstellar Cosmic Ray Spectrum, ArXiv e-prints (Mar., 2013) [arXiv: 1303.6482].

[10] S. Ostapchenko, QGSJET-II: physics, recent improvements, and results for air showers, in European Physical Journal Web of Conferences, vol. 52 of European Physical Journal Web of Conferences, p. 2001, June, 2013.

[11] R. Schlickeiser, Cosmic ray astrophysics. Cosmic ray astrophysics / Reinhard Schlickeiser, Astronomy and Astrophysics Library; Physics and Astronomy Online Library. Berlin: Springer. ISBN 3-540-66465-3, 2002, XV + 519 pp., 2002.

[12] S. Krakau and R. Schlickeiser, Pion Production Momentum Loss of Cosmic Ray Hadrons, ApJ 802 (Apr., 2015) 114.

[13] B. Coste, L. Derome, D. Maurin, and A. Putze, Constraining Galactic cosmic-ray parameters with $Z \leq 2$ nuclei, $A \& A \mathbf{5 3 9}$ (Mar., 2012) A88, [arXiv:1108.4349].

[14] A. W. Strong, E. Orlando, and T. R. Jaffe, The interstellar cosmic-ray electron spectrum from synchrotron radiation and direct measurements, A\&A 534 (Oct., 2011) A54, [arXiv:1108.4822].

[15] E. Orlando and A. Strong, Galactic synchrotron emission with cosmic ray propagation models, MNRAS 436 (Dec., 2013) 2127-2142, [arXiv: 1309.2947 ].

[16] Planck Collaboration, Planck 2015 results. XXV. Diffuse low-frequency Galactic foregrounds, ArXiv e-prints (June, 2015) [arXiv:1506.0666].

[17] R. Kissmann, M. Werner, O. Reimer, and A. W. Strong, Propagation in 3D spiral-arm cosmic-ray source distribution models and secondary particle production using PICARD, Astroparticle Physics 70 (Oct., 2015) 39-53, [arXiv: 1504 . 0824].

[18] R. Kissmann, PICARD: A novel code for the Galactic Cosmic Ray propagation problem, Astroparticle Physics 55 (Mar., 2014) 37-50, [arXiv: 1401.4035 ].

[19] R. Jansson and G. R. Farrar, A New Model of the Galactic Magnetic Field, ApJ 757 (Sept., 2012) 14, [arXiv:1204.3662].

[20] R. Jansson and G. R. Farrar, The Galactic Magnetic Field, ApJLett 761 (Dec., 2012) L11, [arXiv: 1210.7820$]$. 
[21] K. M. Górski, E. Hivon, A. J. Banday, B. D. Wandelt, F. K. Hansen, M. Reinecke, and M. Bartelmann, HEALPix: A Framework for High-Resolution Discretization and Fast Analysis of Data Distributed on the Sphere, ApJ 622 (Apr., 2005) 759-771, [astro-ph/].

[22] D. Maurin, F. Melot, and R. Taillet, A database of charged cosmic rays, A\&A 569 (Sept., 2014) A32, [arXiv:1302.5525].

[23] The Fermi-LAT Collaboration, Fermi Large Area Telescope Third Source Catalog, ArXiv e-prints (Jan., 2015) [arXiv:1501.0200].

[24] D. Gaggero, A. Urbano, M. Valli, and P. Ullio, Gamma-ray sky points to radial gradients in cosmic-ray transport, PRD 91 (Apr., 2015) 083012, [arXiv: 1411.7623].

[25] D. Maurin, A. Putze, and L. Derome, Systematic uncertainties on the cosmic-ray transport parameters. Is it possible to reconcile B/C data with $\delta=1 / 3$ or $\delta=1 / 2$ ?, A\&A 516 (June, 2010) A67, [arXiv: 1001.0553$].$ 